Prácticas y discursos racistas en el contexto educativo. El caso de la Facultad de Ciencias Sociales en San Cristóbal de Las Casas, Chiapas

\title{
Racist Practices and Discourses in the Scholastic Context. The Case of the Social Sciences School in San Cristobal de Las Casas, Chiapas
}

DOI: https://doi.org/10.32870/dse.v0i13.230

\author{
Margarita de Jesús Gutiérrez Narváez*
}

Resumen: El artículo presenta un análisis en relación a las diversas formas de expresión del racismo, entre las que podemos encontrar la discriminación, la exclusión, la violencia física y psicológica, la segregación, el prejuicio, entre otras. Específicamente abordaremos estas manifestaciones racistas a partir de las prácticas y los discursos. El análisis se realizó en la Facultad de Ciencias Sociales, Campus III de la UNACH, en la ciudad de San Cristóbal de Las Casas, tomando en cuenta que es uno de los espacios en donde las expresiones de racismo se presentan en las prácticas cotidianas de socialización e interacción, en los espacios áulicos y en la vida cotidiana del contexto escolar. En este estudio se resalta la naturaleza del racismo sutil que existe en el uso del lenguaje a partir de los diversos discursos racistas expresados por los actores sociales. Palabras clave: racismo, prácticas y discursos racistas, discriminación, exclusión, educación.

\begin{abstract}
This article analyzes different expressions of racism, including discrimination, exclusion, physical violence, segregation and prejudice, among others. Special focus is given to practices and discourses. The research took place in the Social Sciences School, Campus III of the Autonomous University of Chiapas (UNACH) in the city of San Cristobal de Las Casas, Mexico, under the premise that expressions of racism can be found in daily practices of socialization and interaction, in classrooms, and in everyday school activity. The study pays special attention to the nature of the subtle racism found in the use of language in different racist discourses expressed by social actors. Key words: racism, racist discourses and practices, discrimination, exclusion, education.
\end{abstract}

\section{Introducción}

Analizar el racismo no es tarea sencilla, dado que presenta una serie de implicaciones teóricas y metodológicas, pero también por la constante negación de la existencia del mismo, además de la confusión del concepto de racismo como categoría de análisis con otros conceptos, sobre todo con el de discriminación. Por todo lo anterior, se hará un breve recorrido teórico acerca de lo que se entiende por racismo y sus diversas manifestaciones o formas en las que se expresa, como son: la discriminación, el estereotipo, el prejuicio, la violencia, la segregación.

*Doctora en Ciencias Sociales y Humanísticas por el Centro de Estudios Superiores de México y Centroamérica (CESMECA). Miembro del SNI Nivel 1, Profesora de asignatura de la Facultad de Ciencias Sociales de la UNACH, Campus III. Correo electrónico: m-gutierrez-n@hotmail.com 
Las manifestaciones de racismo se expresan tanto de manera explícita en prácticas sociales de rechazo, exclusión y discriminación, mismas que legitiman los mecanismos de dominación social, como de forma sutil en el orden de lo simbólico: los discursos y juegos de lenguaje donde se sobreponen gestos, juicios de valor y desacreditación a partir de estigmas, estereotipos y prejuicios.

Las diversas manifestaciones de racismo se presentan tanto en los espacios públicos como en los privados, en este análisis nos centramos en el contexto escolar, en donde las diversas expresiones de racismo se manifiestan en las relaciones cotidianas de interacción y socialización entre los alumnos y docentes de la facultad.

Para explicar dichas manifestaciones que se presentan en el contexto educativo nos centramos en los discursos y las prácticas racistas de los diversos actores sociales, tanto de docentes como de alumnos de la institución.

\section{Metodología}

Para comprender la dialéctica entre los discursos y las prácticas racistas, en términos metodológicos se realizó un estudio de carácter cualitativo, recurriendo a la operacionalización de los conceptos de racismo, discriminación, prácticas y discursos.

Por otro lado, los discursos y las prácticas racistas de los grupos dominantes se analizaron retomando el planteamiento de Van Dijk (2009), quien señala que el discurso se analiza no solamente como un objeto "verbal" autónomo, sino también como una interacción situada, como una práctica social o como un tipo de comunicación que se da en una situación social, cultural, histórica o política.

El discurso racista de los miembros de endogrupos tiene la peculiaridad de poner énfasis, mediante muchas variantes discursivas, en las características positivas de nuestro propio grupo y de sus miembros y en las características negativas de los otros, el exogrupo. Un aspecto por demás trascendental en la metodología es el análisis de los discursos escritos y hablados como eventos de comunicación y como formas de interacción en el contexto educativo.

Con el propósito de articular el estudio de las prácticas con el análisis de los discursos racistas, se sitúa el trabajo dentro de una perspectiva comprensiva de las ciencias sociales. La etnografía se impone como el método en investigaciones de corte comprensivo, misma que permitió registrar los comportamientos culturalmente significativos de las situaciones a estudiar.

Como parte de las técnicas del enfoque cualitativo, se realizaron 20 entrevistas a profundidad a estudiantes de las cuatro licenciaturas que oferta la Facultad de Ciencias Sociales (Sociología, Economía, Antropología Social e Historia), de los cuales diez fueron estudiantes indígenas y diez estudiantes mestizos o no indígenas. De la misma forma se entrevistó a diez profesores que forman parte de la plantilla académica de la institución. La selección de las personas entrevistadas fue a partir de un acercamiento previo y con la confianza de conocerlos a partir de la convivencia en el espacio educativo. Formar parte de la plantilla de docentes de la carrera en Sociología me permite 
tener un mayor acercamiento con los estudiantes y sobre todo conocer muchas de las problemáticas que se presentan al interior de la institución, entre ellas el grave problema del racismo.

Por otro lado, el quehacer como docente de la institución me permitió realizar observaciones constantes en los espacios áulicos, así como en los diversos espacios en donde los estudiantes socializan, como son la cafetería, la biblioteca, los patios, los pasillos, las áreas deportivas, entre otros.

Queda decir que a todas las personas entrevistadas, tanto estudiantes como docentes, se les informó del uso que se haría de la información obtenida y dado el carácter del tema a tratar y por cuestiones de ética se ha cambiado el nombre de todos los entrevistados.

\section{Acercamiento al contexto}

Los Altos de Chiapas confieren inevitablemente un papel de primera importancia a la ciudad de San Cristóbal de Las Casas, en tanto que es el principal municipio comercial de la región. San Cristóbal de Las Casas, anteriormente conocido como Ciudad Real, se fundó el 31 de marzo de 1528 por el capitán general español Diego de Mazariegos, de igual manera los barrios de San Cristóbal se fundaron en este mismo año; al noroeste de la ciudad quedaron los barrios de Mexicanos y Tlaxcala, a veces considerados como un solo barrio en el que se asentaron varios de los indios mexicas, tlaxcaltecas y tal vez indios de otras regiones de Mesoamérica (Viqueira, 2007: 34).

San Cristóbal de Las Casas es una sociedad multicultural en la que viven personas con diferentes niveles socioeconómicos, es decir, ricas, pobres, indígenas, ladinas; los ladinos ${ }^{1}$ ricos, por lo general, viven en el centro de la ciudad o en algunos barrios, mientras que la mayor parte de los indígenas se encuentran asentados en las colonias periféricas (Calvo, 1991: 55).

La colonial ciudad de San Cristóbal de Las Casas era el centro administrativo de la región y conjunta entre sus habitantes las poblaciones mestizas locales, avecindadas y extranjeras, además de una importante comunidad indígena proveniente de los diferentes municipios de la región y de otras; esta población indígena se ubica principalmente en el margen del periférico de la ciudad. La dinámica económica de la región se basa en el turismo, actividades agropecuarias, una creciente industria de la transformación, comercio y la oferta de servicio a la comunidad (Rodríguez, 2010: 103).

San Cristóbal se ha expandido de una manera acelerada a raíz de la llegada de un numeroso contingente de indígenas de Los Altos en busca de mejores trabajos y condiciones de vida desde principios de los años setenta, en su mayoría expulsados de sus comunidades por problemas religiosos, políticos y económicos. Muchos de ellos procedían de comunidades de Chamula, Oxchuc, Huixtán, Larráinzar y Mitontic; además de llegar en busca de oportunidades de empleo, muchos

1 Ladino no es una categoría racial sino cultural, que denota a los que hablan español, usan el tipo de ropa que se acostumbra en el resto del país y no son indígenas. Esencialmente significa "no indígena" (Estrada, 2009: 170). En la ciudad de San Cristóbal de Las Casas también se les llama mestizos a quienes no son indígenas. 
jóvenes indígenas llegan a la ciudad para poder cursar sus estudios universitarios y la Facultad de Ciencias Sociales es una institución elegida por muchos jóvenes indígenas para realizar sus estudios en el nivel superior.

Para Villafuerte (2010: 121) la composición étnica de la población también ha experimentado cambios dramáticos. En la actualidad podemos observar en San Cristóbal a indígenas, sobre todo tzotziles y tzeltales, provenientes de comunidades cercanas, así como otros llegados de otros estados del país, e incluso de otros países.

San Cristóbal de Las Casas anteriormente era "orgullosamente" criolla, aunque es innegable la relación que tiene la ciudad con las comunidades indígenas de sus alrededores desde hace muchos años, y no sólo en lo comercial, puesto que los indígenas, aparte de llegar a la ciudad a vender sus productos, también mandan a sus hijos a estudiar a las escuelas del nivel medio superior y superior, ya que en la mayoría de las comunidades no tienen ese servicio (París, 1997: 90).

En la actualidad en esta ciudad, las relaciones entre indígenas y mestizos presentan distintas formas de cooperación, de tensión y de conflicto, de simulación u hostilidad, que se expresan en manifestaciones diversas de racismo. Estas manifestaciones en la mayoría de los casos tienen que ver con los procesos de construcción y reproducción de las identidades indígenas y mestizas en la ciudad (Gutiérrez, 2014: 25).

Es indudable que existe una estrecha relación entre la antigua Ciudad Real ${ }^{2}$ y las comunidades indígenas que la rodean. Incontables transacciones comerciales con los pueblos indios constituían el sustento económico principal de la ciudad. Burguete (1998: 129) destacaba, en particular, la venta de las siguientes mercancías: telas, aguardiente, velas, pólvora, sombreros, morrales y alimentos para los indígenas; éstos a su vez vendían en el mercado local sus productos agrícolas y artesanales. Los comerciantes realizaban también labores de mediación entre los poblados tsotziles, comprando y vendiendo sus productos en los mercados. Este proceso de mercantilización sigue presente, aunque se ha modificado la venta de algunas mercancías, como por ejemplo el aguardiente, que ya no se vende libremente en el mercado, y muchos de los indígenas que sólo venían a vender sus productos y regresaban a sus comunidades, hoy han establecido su residencia en la ciudad.

En este sentido se retoma a Remesal cuando ilustra con mucha claridad cómo se han presentado las relaciones entre estos dos grupos sociales, mestizos e indígenas:

en San Cristóbal de Las Casas, orgulloso bastión de los coletos, los indios se bajaban de las aceras para ceder paso a los blancos, que seguían refiriéndose a ellos con un asco racial, en el que resonaba el eco de la frase brutal, que escucho fray Bartolome a su llegada a la ciudad, «perros indios» (Remesal, 1988: 447).

2 Ciudad Real es uno de los diversos nombres que se le han dado a la actual ciudad de San Cristóbal de Las Casas. 
En las relaciones entre indígenas y mestizos en la ciudad siempre han estado presentes los procesos de dominación en medio de una serie de conflictos, enfrentamientos y crisis; entre ellos el surgimiento del Ejército Zapatista de Liberación Nacional. Al respecto Arellano y Santoyo señalan que:

Históricamente, en los procesos de dominación han surgido actores, movimientos y sujetos sociales que se han opuesto y resistido a los mismos. En el año de 1994, la sociedad marca el tiempo de la emergencia de lo que podría considerarse un nuevo sujeto social, quien además de las necesidades básicas, plantea el respeto a la dignidad de la población indígena como forma de conciencia histórica de su quehacer social, personal o de lucha; sujeto que, como forma de expresión social, sintetiza y articula a través de una organicidad, la oposición real o ideológica a formas de explotación inhumanas, a formas de discriminación social expresadas en las más lamentables condiciones de vida y el trato humillante a que son sometidos los indígenas del país, especialmente los del sureste mexicano (Arellano y Santoyo, 1997: 142).

Aunado a lo anterior podemos señalar que a partir de 1994 la convivencia entre mestizos e indígenas urbanizados -emigrados o expulsados de distintos municipios alteños, ahora residentes en las colonias periféricas de la ciudad- se ha intensificado. Como las condiciones económicas y políticas por las cuales los estaban expulsando de sus lugares tradicionales seguían vivas desde los años setenta, el crecimiento de la población indígena de la ciudad no se detuvo, y después se aceleró durante la década de 1980. En 1990, el municipio de San Cristóbal ya contaba con 90000 habitantes, y quizá unos 20000 eran indígenas. En el año 2000, el conteo oficial de toda la población alcanzó 120 000, mientras otros cálculos estadísticos daban la cifra de 160 000, un aumento de entre 460 y $570 \%$ en sólo treinta años, de esta cifra, aproximadamente 60000 eran indígenas (Rus, 2009: 182).

La escenografía que configura actualmente la ciudad de San Cristóbal, en cuanto a presencia de población indígena, es bastante distinta a la de fines de la década de 1960 y principios de la década de 1970. Hasta esas fechas, las condiciones de las relaciones económicas y sociales de la urbe limitaban a la población indígena a no radicar en la ciudad, salvo para situaciones de algún servicio público.

El crecimiento poblacional en la ciudad ha sido un factor determinante para poder explicar las relaciones, en la mayoría de los casos de conflicto, entre indígenas y coletos, ${ }^{3}$ ya que muchos atribuyen a la llegada masiva de los indígenas al valle de Jovel la causa principal de todos los problemas que tiene la ciudad.

3 El término coleto es una referencia y auto referencia de los habitantes de San Cristóbal de Las Casas, en particular de las familias mestizas que llevan varias generaciones viviendo en la ciudad (París, 1997: 91). La identidad coleta es la que ha prestado gran importancia al aspecto educativo como un elemento diferenciador del resto de las clasificaciones que interactúan en la ciudad de San Cristóbal de Las Casas, esta característica de definirse como un grupo "culto", que "aunque sean licenciados”, es típica de las noblezas que son esencialistas (Sulca, 1999: 65). Muchas personas señalan que la identidad coleta finca sus raíces en "la nobleza", en la educación, mientras que otros ubican su identidad a partir de la diferencia (Gutiérrez, 2014: 100). 
Los mestizos de San Cristóbal durante la mayor parte de esos años aplicaron barreras excluyentes y racistas dentro de la ciudad, con la intención de proteger su precaria posición como explotadores de la población indígena, que al mismo tiempo que los rodeaba, los superaba en número. Dichas barreras hicieron de la ciudad un espacio privilegiado para los mestizos, y extremadamente hostil para los indígenas.

Lo que hace de San Cristóbal un caso aparte, empero, es que si bien históricamente la ciudad ha sido un lugar ladino, orgulloso de sus tradiciones "españolas" y hostil a la población indígena, todos los migrantes recientes son mayas provenientes de los poblados vecinos (Rus, 2009: 170).

Actualmente gran parte de la población indígena radicada en la ciudad ya cuenta con mejores condiciones de vida; muchos de los que llegaron como migrantes a la ciudad ahora ya están totalmente establecidos y sus hijos asisten a las escuelas como todos los demás.

Es en este contexto de relaciones interétnicas en donde se funda la Facultad de Ciencias Sociales de la Universidad Autónoma de Chiapas (UNACH), que fue inaugurada el 17 de abril de 1975, con la presencia del presidente de la república Luis Echeverría, quedando como rector de la misma Federico Salazar Narváez (Santiago, 2004).

La UNACH es una institución pública de carácter universitario para la educación superior, cuya sede está en la ciudad de Tuxtla Gutiérrez, capital del estado de Chiapas; cuenta con centros académicos en Arriaga, Comitán, Pichucalco, San Cristóbal de las Casas, Palenque, Copainalá, Tapachula y Villaflores, Huehuetan y Pijijiapan. La conforman nueve campus en el estado de Chiapas, y es en el Campus III donde se encuentra la Facultad de Ciencias Sociales.

De acuerdo con Gordillo (1988), miembro de la Academia de Sociología del Campus III, UNACH, la Facultad de Ciencias Sociales inició sus actividades en 1977 en la ciudad de San Cristóbal de Las Casas, Chiapas, ofreciendo las carreras en Economía, Sociología, Historia y Antropología Social. Se ofrecía también la carrera en Administración Pública, pero se eliminó por no tener el número de alumnos necesarios para mantenerla.

A la Facultad de Ciencias Sociales ingresan estudiantes indígenas y mestizos, tanto radicados en la ciudad como habitantes de las comunidades cercanas. La elección de carrera se da por intereses personales y es en las cuatro licenciaturas en donde podemos encontrar a estudiantes tzotziles, tzeltales, choles, tojolabales y mestizos. Incluso por la dinámica institucional de intercambios académicos, esta facultad recibe a muchos estudiantes extranjeros.

\section{Recorrido teórico}

El estudio del racismo ha sido analizado desde varias miradas tanto teóricas como metodológicas, es importante señalar que para esta investigación entendemos por racismo, de acuerdo a lo que plantea Memmi (1993: 23), la valoración generalizada y definitiva de unas diferencias, biológicas o culturales, reales o imaginarias, en provecho de un grupo y en detrimento del otro, con el fin de justificar una agresión y un sistema de dominación. 
Gómez (2005: 11) señala que el racismo se presenta como un amplio sistema de pensamiento y modo de percibir las diferencias con el otro, el cual se presenta en aquellos humanos inferiores, que les sirve para constatar su propia excelencia y superioridad. Es decir, que el racismo es un conjunto de ideas diferencialistas que tiene un determinado grupo hacia otro, estas ideas son aquellos pensamientos tales como el prejuicio y el estereotipo, que al mismo tiempo generan divisiones sociales y marcan límites fronterizos entre una cultura y otra (Simmel, 2000).

En este sentido, Lewis (1962: 126) enfatiza que el racismo es un ejemplo más de persecución de las minorías en beneficio de quienes detentan el poder. Es decir, que lo define desde una perspectiva ideológica dado que el grupo que tiene el poder manifiesta hacia el grupo minoritario una ideología de superioridad, que les sirve a éste para subordinar, controlar y manipular al grupo pequeño.

Por su parte, Wieviorka (1992: 17) señala que el racismo es sinónimo de exclusión o de rechazo de la alteridad. Es decir, que es la expresión de eliminación o supresión que tiene un grupo social hacia otro grupo, expresiones que se dan a partir de la no aceptación de la alteridad social de determinado grupo; por lo tanto, cuando su modo de vida se ve amenazado, los integrantes actúan de manera negativa hacia el grupo externo.

Por otro lado, para Warman el racismo implica sentimientos de lástima hacia el indígena, a diferencia de otros autores que definen el racismo con sentimientos de odio y miedo hacia éstos, por lo tanto, señala:

Por racismo me refiero al conjunto de prejuicios que percibe diferencias físicas en los indígenas y las relaciona con capacidades disminuidas o diferentes con un origen hereditario. ...Los sentimientos racistas se expresan más como lástima que como odio o temor. Hacen referencia a los pobrecitos, a los inditos, diminutivos ofensivos que expresan su superioridad (Warman, 2003: 84).

Por lo que se ha señalado anteriormente, el racismo es definido y conocido como una ideología de dominio y superioridad de un grupo sobre otro, para el caso del contexto chiapaneco y específicamente en la ciudad de San Cristóbal de Las Casas, una ideología de dominio de los mestizos sobre los indígenas.

Ya presentamos algunas de las discusiones teóricas en relación con el concepto de racismo, ahora presentamos algunas de sus manifestaciones o expresiones más comunes. Entre ellas está la discriminación; generalmente se entiende por discriminación cuando una persona o grupo exterioriza una actitud negativa (excluir o despreciar) a otros que no pertenecen a su mismo grupo, esta actitud suele basarse en las diferentes características físicas o culturales.

La discriminación se refiere en efecto, a la actitud negativa que se manifiesta hacia un grupo social y sus componentes. Es decir, que la discriminación es un modo de expresión que tiene un grupo hacia otro. Se entiende que la actitud negativa hacia un grupo social surge a partir de los elementos que componen cada 
cultura, por ejemplo, la cultura mestiza y la cultura indígena no comparten las mismas tradiciones, por lo tanto, esto genera divisiones sociales entre ambos grupos (Zanfrini, 2007: 105).

Cabe señalar que la discriminación según Zanfrini (2007: 108) es un fenómeno empíricamente observable, que persiste a pesar de las medidas en su contra y que no sólo afecta a los inmigrantes de la primera generación, sino con mucha frecuencia a sus descendientes, sobre todo cuando tiene marcadores étnicos y pertenecen a grupos negativamente estigmatizados.

La segregación es otra práctica racista, que generalmente se da cuando surge un alejamiento físico geográfico de un determinado grupo del resto de la población. Para Wieviorka (1992: 129) es segregación cuando se mantiene al grupo racializado a distancia y se le reserva espacios propios, que únicamente puede abandonar en determinadas condiciones, más o menos restrictivas. Es decir, que la segregación se da a través de la exclusión de espacios físicos, algunos elementos para dar paso a la segregación son las ideas dominantes que tiene cierto grupo hacia otro, cabe mencionar que las ideas se dan a partir de no querer compartir el espacio geográfico.

Muchas son las formas o manifestaciones de racismo, pero en este artículo no pretendemos hacer un análisis de todas ellas, sólo presentamos algunas de las manifestaciones que se presentan de forma más recurrente en la Facultad de Ciencias Sociales.

\section{Prácticas y discursos racistas en el contexto educativo}

Los discursos racistas se presentan en todos los contextos, pero para esta investigación nos interesan primordialmente los que se dan en los contextos educativos. En este sentido Van Dijk (2003) señala que el racismo (así como el antirracismo) puede manifestarse en todo tipo de discurso y de práctica social circunscritos a la educación y a la investigación, como pueden ser las lecciones o la interacción oral entre profesores y alumnos, el programa de estudios oficial, los informes de laboratorio, las notas de campo y las publicaciones científicas.

Fue en la última década del siglo pasado cuando surgió una mayor preocupación por los discursos que expresan prácticas sociales discriminatorias, entre las que destacan, especialmente, las diferentes formas de reproducción cotidiana del racismo en el complejo proceso de comunicación pública e interpersonal (Van Dijk, 2007: 86). Este tipo de discursos, como expresión, confirmación o legitimación de opiniones, actitudes e ideologías del grupo social o étnico dominante, contribuye a la reproducción del racismo y de las formas de dominación vigentes (Gutiérrez, 2014: 65).

Entre los aspectos que deben analizarse cuando se estudian las manifestaciones racistas está el de las prácticas. Retomamos la idea de Memmi (1993), quien señala que en las prácticas racistas siempre van a estar presentes las diferencias, que pueden ser objetivas, como el género, el color de piel, el lugar de origen o el nivel económico, y las subjetivas, que son las más frecuentes y entre las que se incluye el sentimiento de superioridad, la inteligencia, el sentido de pertenencia, los estereotipos o los prejuicios. 


\section{Prácticas de inclusión y exclusión}

Uno de los temas más importantes en cualquier agenda de estudios sobre las relaciones interculturales es el del reconocimiento y la exclusión, tal vez sería más adecuado decir reconocimiento o exclusión (Alcalá, 2008: 9).

En la convivencia cotidiana entre los alumnos de la Facultad de Ciencias Sociales se observaron situaciones de exclusión, principalmente en los salones de clases y en los espacios de socialización. Al entrevistar a estudiantes indígenas y preguntarles si en algún momento se han sentido excluidos por sus compañeros mestizos, muchos de ellos comentaron que sí se sienten excluidos por su vestimenta o su lenguaje, y esto hace que no socialicen con todos sus compañeros de clase e incluso que no pregunten cuando tienen dudas, porque se sienten mal cuando sus compañeros se burlan de la forma en como hablan.

Sí, me siento mal, a veces con los comentarios que se hacen dentro del salón de clases por personas que por no ser indígenas se sienten superiores a ti, me he sentido excluida cuando a veces los compañeros no quieren trabajar conmigo o tiene ciertos comentarios, como cuando te preguntan de dónde eres o no te aburres en tu pueblo, que aburrido sería vivir ahí, son cosas que no entiendes realmente por qué lo hacen. ${ }^{4}$

El sentido de superioridad es uno de los factores predominantes de las personas racistas, siempre se considera que el grupo al que se pertenece es el mejor y "los otros" son los que no saben, a los que no les llega la modernidad, como le señalan a Olga al preguntarle si no se aburre en su pueblo.

De acuerdo con Méndez (2006: 2) la exclusión social se entiende generalmente como el apartamiento de grupos de individuos - por razones diversas, aunque fundamentalmente económicasde la corriente principal de la sociedad.

Bueno, me siento mejor relacionarme con personas con las cuales tengo más cosas en común. Me relaciono con personas que son como yo, tanto en la apariencia física o que se vistan como yo, con personas que vengan de la misma comunidad de donde vengo yo o puedan hablar mi lengua para que me entiendan y no se burlen de mí, porque yo siento que los que son de mi cultura, que son indígenas son más sencillos, porque los demás no nos hablan, prefiero llevarme con gente indígena, porque es como de mi casa. ${ }^{5}$

En la sociedad moderna las personas pertenecen a tantos grupos que algunas de sus relaciones de pertenencia pueden sobreponerse. Horton y Hunt (1998: 201) señalan que la exclusión de un grupo de pertenencia puede ser un proceso brutal y citan como ejemplo que en las sociedades

4 Entrevista realizada a Olga, estudiante indígena de la carrera de sociología, originaria de San Andrés Larráinzar. Abril de 2016

5 Entrevista realizada a Micaela estudiante indígena, de la carrera de Sociología, originaria de Huixtán. Abril de 2016. 
primitivas trataban a los extraños como parte del reino animal: muchos no tenían palabras distintas para referirse a "enemigos" y "extranjeros", con lo que mostraban que no hacían distinción entre ellos. En este tipo de exclusiones o negaciones de la alteridad puede verse claramente cómo se presentan hechos de violencia simbólica.

\section{Relaciones entre mestizos e indígenas}

En cuanto a las formas de socialización entre estudiantes indígenas y mestizos, observamos que cada grupo se reúne o se identifica con las personas que considera son de su propio entorno. El proceso de identificación por oposición al otro ha existido siempre en la historia. Por ejemplo, los griegos antiguos dividían el mundo entre griegos y bárbaros, siendo los bárbaros los que no podían hablar griego, los que balbuceaban o mascullaban otras lenguas, aquellos con los que es difícil comunicarse y entenderse, por lo que se convertían así en los "otros" (Gutiérrez, 2014: 91).

No es que no quiera llevarme o tener amigos indígenas, sí les hablo, pero la verdad me desespera porque no les entiendo, son dos mundos diferentes, dos culturas diferentes, ellos no entienden bien, llegan a la Facultad sin saber prepararse, creo yo, porque les cuesta expresarse de manera adecuada, no hablan bien español o a veces cuando leemos textos complicados de autores difíciles, ellos no entienden y yo la mera verdad no tengo paciencia, ni ganas de enseñarles. Creo que deben venir mejor preparados y aprender bien español antes de entrar a la escuela o de plano que se vayan a una escuela bilingüe. ${ }^{6}$

Podemos ver en los comentarios de Carlos que el idioma juega un papel primordial y hace que llegue a discriminar a los estudiantes indígenas, dado que él considera que no hablan bien el español, en este testimonio se visualizan varias prácticas de racismo, como la discriminación por el idioma, así como la exclusión al desear que se vayan a otra escuela; también se presentan prejuicios relacionados a los procesos de aprendizaje, comúnmente se ha considerado al indígena como el atrasado o ignorante. Al igual que Carlos, podemos ver en los comentarios de Adrián manifestaciones racistas.

Desde mi punto de vista, no es igual la relación que existe entre estos tipos de personas, porque los jóvenes indígenas pueden llegar a ser más tímidos, retraídos, aún no entienden la cultura o la forma de vida que se lleva en un ambiente más grande, en donde la tecnología tiene un mejor avance y eso trae ciertas desventajas en ellos, porque no están familiarizados, no lo entienden, no saben ni encender una computadora, porque en su pueblo nunca lo habían visto. Los jóvenes que somos de la ciudad estamos más al alcance de la tecnología, por lo mismo buscamos amistades que sean bien aceptadas en nuestra sociedad. De verdad

6 Entrevista realizada a Carlos, estudiante mestizo, de la carrera de Economía, originario de San Cristóbal de Las Casas. Abril de 2016. 
no es porque no quiera, pero si te ven que te juntas con un indígena, luego luego te critican. Yo creo que deberían de abrir más universidades como la UNICH, pero sólo en sus pueblos, que no vengan a la ciudad. ${ }^{7}$

El caso de Adrián es clara manifestación de segregación y el grado de desconocimiento hace que su discurso racista sea de mayor peso, dado que considera que la UNICH es sólo para indígenas, desconoce claramente que es una universidad intercultural.

Por tanto, la segregación es una manifestación concreta del racismo, los procesos de segregación fijan distancias morales que convierten los espacios en pequeños mundos que se rozan sin interpretarse (Wieviorka, 1991: 134)

\section{Discriminación}

La discriminación se materializa en casos de humillaciones, malos tratos, desigualdad de oportunidades o burlas, así como en pobreza y dificultad de acceso a poder tener una vida adecuada. De acuerdo con el Consejo Nacional para Prevenir la Discriminación (CONAPRED), la discriminación es una práctica que consiste en dar un trato favorable o de desprecio inmerecido a determinada persona o grupo, que a veces no percibimos, pero que en algún momento la hemos causado o recibido.

En este sentido son muchas las prácticas de discriminación que se dan en el contexto escolar, específicamente en la Facultad de Ciencias Sociales. De acuerdo con los datos obtenidos en el departamento de servicios escolares de la institución, $40 \%$ de la población estudiantil es de origen indígena, en tanto que $60 \%$ son mestizos. Es importante destacar que, en el contexto de la vida cotidiana de la facultad, los estudiantes indígenas no visten sus trajes regionales, en muy raras ocasiones las estudiantes portan alguna blusa bordada que representa su comunidad de origen, pero por lo general los alumnos visten ropa casual.

Fue algo que me enojó mucho, ya que una compañera tenía unas amigas y ese día fuimos juntas todas a desayunar a la cafetería, entonces paso una chava del lugar de donde yo vengo con una de sus amigas y comenzaron hablar muy feo de ella, que era una india que no sabía vestirse y que la ropa que tenía no le quedaba, ni mucho menos el maquillaje y una de las chavas dijo, "pobre india, tenía que ser de Zinacantán". 8

Podemos ver cómo se presenta la discriminación, como una manifestación de racismo, al menospreciar la cultura de alguna persona, en este caso el de Lucía, señalando que por ser de Zinacantán, una comunidad indígena de los Altos de Chiapas, no puede vestirse o arreglarse como ella quiera, y se usa el término "india" en un tono despectivo.

7 Entrevista realizada a Adrián, estudiante mestizo, de la carrera de Historia, originario de San Cristóbal de Las Casas. Abril de 2016.

8 Entrevista realizada a Lucía, estudiante indígena de la carrera de Economía, originaria de Zinacantán. Abril de 2016. 
Se discrimina por el lugar de origen, por el idioma, por la forma de vestir, por las diferencias culturales tal como lo señala Claudia.

Creo que me ven mal por mi lengua, me limita porque no domino bien el español, porque cuando estoy en mi casa todos hablamos mi lengua, nadie habla el español y no lo domino bien, ya que estoy más acostumbrada a mi lengua y no sé cómo decir algunas cosas, y si las digo mal me corrigen. También me siento mal cuando me miran si traigo mi traje de la comunidad, por eso aquí en la escuela no lo uso, sólo en mi casa, porque siento que me ven feo y escuchas como te critican con voz baja. Cuando participo en clase y hablo de mi cultura, de mi comunidad, mis compañeros siempre hacen burla, gestos y no les interesa lo que digo. ${ }^{9}$

Los inmigrantes indígenas una vez instalados en las ciudades lidian con el rechazo propiciado por los estereotipos con que la cultura urbana los concibe. Cotidianamente chocan con la incomprensión de las instituciones y los espacios públicos respecto al valor de su lengua y de sus costumbres, unas instituciones renuentes a reconocer las diferencias y a corregir las desigualdades (García, 2005: 34).

La discriminación por la lengua es una de las prácticas racistas más frecuente en la facultad, tanto los jóvenes estudiantes como algunos docentes se burlan de los jóvenes indígenas cuando no pronuncian bien alguna palabra.

Realmente es muy difícil el trabajo como docente aquí en la facultad cuando tenemos alumnos indígenas, sobre todo en el turno de la tarde, en la mañana no tengo problemas, pero los chicos de la tarde sí se me complican mucho. No saben leer, no entienden las lecturas y cuando exponen no se les entiende nada. Lo que hago es aprobarlos, porque pobrecitos no creo que lleguen a entender, y cuando les toca exponer a los alumnos indígenas, trato de darles lecturas pequeñas y aun así les cuesta mucho. Por eso ni siquiera asesoro para tesis, porque pobres, cómo van a llegar a una discusión teórica. ${ }^{10}$

Podemos ver en algunos docentes y en muchas de las prácticas institucionalizadas, ${ }^{11}$ que existe un paternalismo disfrazado de racismo; se les dice a los indígenas "pobrecitos", "inditos", "chamulitas", aludiendo al término diminutivo; se les hace menos, siempre se piensa que son atrasados, que no entienden. Esto es lo que Van Dijk (2007) llama racismo cotidiano, son los tipos de discurso e interacción verbal cuya utilización normalmente se considera aceptable en las conversaciones o en las acciones del grupo dominante.

9 Entrevista realizada a Francisco, estudiante indígena de la carrea de Economía, originario de Oxchuc. Abril de 2016.

10 Entrevista realizada a Carmen, docente de la Facultad de Ciencias Sociales. Abril 2016.

11 Las prácticas institucionalizadas son aquellas que legitimadas por la cultura organizacional, refieren al conjunto de normas y valores que las sustentan como parte de la sociedad humana en general; en el contexto académico pueden señalarse como prácticas institucionalizadas, las lecciones, los libros de texto, los exámenes, las asignaturas, las correcciones, los actos de grado, entre otras (Van Dijk, 2007). 
Las lenguas indígenas son vistas todavía como atrasadas y primitivas; el español, por el contrario, tiene valor simbólico como lengua de civilización, progreso y modernidad, además de un alcance instrumental como medio de sobrevivencia en la sociedad dominante y de movilidad socioeconómica. Los hablantes monolingües de las lenguas indígenas son vistos como portadores de una deficiencia lingüística que sólo puede remediarse a través de la castellanización (Freedson y Pérez, 1999: 60).

Yo no participo en clase porque me da mucho miedo hablar y participar, me pasa esto con varias clases, muchas veces no entiendo lo que dicen los maestros y no pregunto porque siempre se burlan de mi forma de hablar, si digo una palabra mal, escucho sus risas. La maestra que me da investigación me dijo un día, "ya no te revisaré más, ya me cansé, no te entiendo, ve a aprender español", ese día ya no quería regresar a la escuela, pero mis amigos me convencieron de que siguiera. Pienso que por eso saco bajas calificaciones. ${ }^{12}$

Los espacios fundamentales en donde se reproduce el racismo son principalmente en la familia y en la escuela, siendo los padres y maestros los principales agentes reproductores de las prácticas y discursos racistas. La familia es la primera institución de socialización, y la escuela es el contexto en donde se viven muchos aspectos de la vida cotidiana.

El sistema racista, señala Van Dijk (1994), consta de la acción social, acciones discriminatorias desfavorables para otros y de la cognición social, modelos mentales, conocimientos, actitudes, ideologías, normas y valores que son compartidos por los dominantes. La existencia de este sistema depende de su reproducción cotidiana a través del discurso del grupo dominante. Como lo podemos observar en el testimonio de Luis, a quien la profesora mando a estudiar español aludiendo a que no le entendía, más que una práctica o estrategia pedagógica, se trata de una práctica y un discurso racista por parte de quien se considera estar en una posición superior.

Coincidimos con Montemayor (2003: 30) cuando señala que una lengua da identidad cuando se sustenta en una comunidad de intereses o valores compartidos por todos, no cuando está impuesta por grupos o regiones que parten de los mismos contextos. En este sentido la lengua española, señala Montemayor, no es una garantía de identidad en todos los pueblos que viven, han vivido o se han desarrollado bajo ese patrón lingüístico.

\section{Conclusiones}

Las prácticas y discursos racistas presentados en este análisis permiten una aproximación de las características de las manifestaciones racistas en el contexto escolar y sobre todo en un contexto universitario. 
Es claro ver, a partir del análisis, que las manifestaciones racistas se presentan en todos los espacios, sean públicos o privados, pero es realmente lamentable que en instituciones educativas se presenten casos de racismo y más lamentable es que sean los profesores quienes tienen estas prácticas racistas.

El sentido equivocado de superioridad que tienen algunos docentes hace que sus discursos racistas dañen a los estudiantes. Como hemos mencionado en muchas de las definiciones de racismo, éste se entiende por el sentido de dominación de un grupo sobre otro. Y es en el contexto escolar en donde algunos profesores consideran que tienen un dominio sobre los estudiantes.

De la misma forma es alarmante que entre estudiantes se dé la discriminación por cuestiones culturales, suponemos que es en las instituciones educativas en donde debemos enseñar el respeto por la diversidad, pero es donde el racismo sutil se presenta en muchas situaciones cotidianas.

Los elementos culturales que no son reconocidos por muchos mestizos, tanto alumnos como docentes en el contexto de la Facultad de Ciencias Sociales, son principalmente el idioma y la vestimenta, mismos que son objeto de burla y rechazo hacia los indígenas.

Finalmente puedo señalar que es urgente presentar propuestas que permitan la concientización en los estudiantes y los docentes para erradicar el racismo de las escuelas, de los hogares, en fin, de todos los espacios.

\section{Bibliografía}

Alcalá Campos, Raúl. (2008). Reconocimiento y exclusión. México: UNAM/Plaza y Valdés.

Arellano Sánchez, José y Margarita Santoyo R. 1997. "Racismo y nuevos sujetos sociales en Chiapas. El surgimiento del EZLN”. En: Cuadernos. VI Jornadas Lascasianas. La problemática del racismo en los umbrales del siglo XXI. México: UNAM. pp. 171-184.

Burguete Estrada, Manuel (1998). Los mercados de San Cristóbal, Merposur: el último del siglo XX, H. Ayuntamiento Municipal de San Cristóbal de Las Casas 1996-1998. San Cristóbal de Las Casas, Chiapas, México.

Calvo Sánchez, Angelino (1991). "Las colonias nuevas de migrantes y expulsados en San Cristóbal de Las Casas". En: Anuario CEI III. San Cristóbal de Las Casas: UNACH. Centro de Estudios Indígenas.

Estrada Saavedra, Marco (2009). Chiapas después de la tormenta. Estudios sobre economía, sociedad y política. México: El Colegio de México.

Freedson González, Margaret y Elías Pérez Pérez (1999). La educación bilingüe bicultural en los Altos de Chiapas. Una evaluación. México: SEP.

García Canclini, Néstor. (2005). Diferentes, desiguales y desconectados. Mapas de la interculturalidad. Barcelona: Gedisa. 
Prácticas y discursos racistas en el contexto educativo. El caso de la Facultad de Ciencias Sociales en San Cristóbal de Las Casas, Chiapas

Gómez Izquierdo, José Jorge (coord.) (2005). Los caminos del racismo en México. México: Plaza y Valdés.

Gordillo Guillén, Oscar (1988). “La sociología en la Universidad Autónoma de Chiapas”. En: Encuentro Nacional de Escuelas de Sociología. Tlaxcala: Universidad Autónoma de Tlaxcala.

Gutiérrez Narváez, Margarita de Jesús. (2014). "Identidad, racismo y familia en San Cristóbal de Las Casas". Colección Thesis. Chiapas: UNICACH/CESMECA.

Horton, Paul B. y Chester L. Hunt (1998). Sociología. México: Mc Graw-Hill.

Lewis, John. (1962). Hombre y evolución. México: Grijalbo.

Memmi, Albert. (1993). Racismo y odio del otro. Madrid: Edicusa.

Méndez, A. (2006). La exclusión social: análisis y propuestas para su intervención. Madrid: Fundación alternativas.

Montemayor, Carlos (2003). "La afirmación de la identidad en el exilio". En: Valenzuela Arce, José M. (coord.). Renacerá la palabra. Identidades y dialogo intercultural. México: COLEF.

París Pombo, María Dolores. (1997). "Identidades colectivas de las elites en el centro de Chiapas (1971-1993): Tuxtla Gutiérrez y San Cristóbal de Las Casas". Tesis doctoral. México: FLACSO, pp. 89-100.

Remesal, Fray Antonio de (1988). Historia general de las Indias Occidentales y particular de la gobernación de Chiapa y Guatemala. Vol. 2. México: Porrúa.

Rodríguez, G., G. Sánchez y L. Zaragoza (2010). "Indígenas establecidos en la periferia de San Cristóbal de Las Casas. Actividades agropecuarias como parte del sistema de vida". En: Anuario de Estudios Indígenas XIV. Migraciones, ciudades y cambio cultural. Chiapas: UNACH, Instituto de Estudios Indígenas.

Rus, Jan (2009). "La nueva ciudad maya en el valle de Jovel: urbanización acelerada, juventud indígena y comunidad en San Cristóbal de Las Casas". En: Estrada Saavedra, Marco (ed.). Chiapas después de la tormenta. Estudio sobre economía, sociedad y política. México: El Colegio de México/Gobierno del Estado de Chiapas/Cámara de Diputados LX Legislatura.

Santiago García, Rosana. (2004). Plan de Estudios de la licenciatura en Sociología. Facultad de Ciencias Sociales de la Universidad Autónoma de Chiapas. México: UNACH.

Simmel, G. (2000). "El conflicto de la cultura moderna". En: Revista Española de investigaciones sociológicas, núm. 89. España: Centro de investigaciones sociológicas, pp. 315-330.

Sulca Báez, Edgar (1999). "Nosotros los coletos. Identidad y cambio en San Cristóbal de Las Casas, Chiapas". Anuario 1996. SEPARATA.

Van Dijk, Teun A. (1994). Racismo y discurso de las élites. España: Gedisa.

— (2003). Dominación étnica y racismo discursivo en España y América Latina. España: Gedisa.

(2007). Racismo y discurso en América Latina. Barcelona: Gedisa. (2009). Discurso y poder. España: Gedisa. 
Villafuerte, D., E. López y J. Molina (2010). “Apuntes sobre las nuevas migraciones en San Cristóbal de Las Casas, Chiapas". En: Anuario de Estudios Indígenas XIV. Migraciones, ciudades y cambio cultural. Chiapas: UNACH/Instituto de Estudios Indígenas.

Viqueira, Juan Pedro (2007). "Historia critíca de los barrios de Ciudad Real”. En: Camacho, D., A. Lomelí y P. Hernández. La ciudad de San Cristóbal de Las Casas, a sus 476 años. Una mirada desde las ciencias sociales. Tuxtla Gutiérrez: Gobierno del Estado.

Warman, Arturo (2003). Los indios mexicanos en el umbral del milenio. México: Fondo de Cultura Económica.

Wieviorka, Michel (1992). El espacio del racismo. Barcelona: Paidós.

Zanfrini, Laura (2007). La convivencia interétnica. Madrid: Alianza.

Recibido: 04/05/16

Dictaminado: 11/07/16

Corregido: 23/09/16

Aceptado: 25/10/16 